Revue internationale P.M.E.

Économie et gestion de la petite et moyenne entreprise

\title{
Le rôle des synergies locales dans les processus spatiaux d'innovation
}

\section{Remingio Ratti}

Volume 4, numéro 3, 1991

URI : https://id.erudit.org/iderudit/1008071ar

DOI : https://doi.org/10.7202/1008071ar

Aller au sommaire du numéro

Éditeur(s)

Presses de l’Université du Québec

ISSN

0776-5436 (imprimé)

1918-9699 (numérique)

Découvrir la revue

Citer cette note

Ratti, R. (1991). Le rôle des synergies locales dans les processus spatiaux d'innovation. Revue internationale P.M.E., 4(3), 77-94.

https://doi.org/10.7202/1008071ar

\section{Résumé de l'article}

Cet article porte sur l'évolution du rapport local-international selon les différents modèles (ou théories) de la diffusion spatiale de l'innovation: les modèles épidémico-hiérarchiques, les modèles de la division spatiale du travail, les modèles réticulaires. Il essaye, d'une façon originale, de situer la position de la PME innovatrice en distinguant trois espaces de vie de la firme : l'espace de marché, l'espace de production et l'espace de soutien.

Les résultats de l'étude montrent que, dans une vision classique, la PME agirait plutôt selon le cadre défini par son espace de marché et dans un modèle où les règles du jeu seraient dictées par le marché et par la hiérarchie urbaine. Dans un contexte structurel de division spatiale du travail, au contraire, la PME devra surtout agir pour adapter son " espace de production " sans pour autant s'affranchir d'une certaine subordination par rapport à la grande entreprise multinationale et aux centres métropolitains.

Enfin, le modèle récent d'analyse selon une approche réticulaire place théoriquement la PME dans une position d'affranchissement par rapport aux subordinations citées, à la condition qu'elle sache développer son espace de " soutien », c'est-à-dire une stratégie de rapport « hors marché » à caractère précompétitif. Or, ce qui est intéressant, c'est que cet espace de soutien sera avec une bonne probabilité réalisable dans certains lieux spatialement marqués par une cohésion nouvelle, par des synergies locales qui représentent à la fois un élément constitutif et un résultat de l'existence d'un « milieu innovateur ", selon la terminologie du GREMI.
Ce document est protégé par la loi sur le droit d'auteur. L'utilisation des services d'Érudit (y compris la reproduction) est assujettie à sa politique d'utilisation que vous pouvez consulter en ligne.

https://apropos.erudit.org/fr/usagers/politique-dutilisation/ 


\title{
Le rôle des synergies locales dans les processus spatiaux d'innovation
}

\author{
Remingio RATTI \\ Université de Fribourg et \\ Instituto di Ricerche Economiche*
}

\begin{abstract}
RÉSUMÉ
Cet article porte sur l'évolution du rapport local-international selon les différents modèles (ou théories) de la diffusion spatiale de l'innovation: les modèles épidémico-hiérarchiques, les modèles de la division spatiale du travail, les modèles réticulaires. II essaye, d'une façon originale, de situer la position de la PME innovatrice en distinguant trois espaces de vie de la firme: l'espace de marché, l'espace de production et l'espace de soutien.
\end{abstract}

Les résultats de l'étude montrent que, dans une vision classique, la PME agirait plutôt selon le cadre défini par son espace de marché et dans un modèle où les règles du jeu seraient dictées par le marché et par la hiérarchie urbaine. Dans un contexte structurel de division spatiale du travail, au contraire, la PME devra surtout agir pour adapter son * espace de production " sans pour autant s'affranchir d'une certaine subordination par rapport à la grande entreprise multinationale et aux centres métropolitains.

Enfin, le modèle récent d'analyse selon une approche réticulaire place théoriquement la PME dans une position d'affranchissement par rapport aux subordinations citées, à la condition qu'elle sache développer son espace de " soutien ", c'est-à-dire une stratégie de rapport " hors marché " à caractère précompétitif. Or, ce qui est intéressant, c'est que cet espace de soutien sera avec une bonne probabilité réalisable dans certains lieux spatialement marqués par une cohésion nouvelle, par des synergies locales qui représentent à la fois un élément constitutif et un résultat de l'existence d'un "milieu innovateur », selon la terminologie du GREMI.

* Remingio Ratti est professeur d'économie régionale et d'économie des transports à l'Université de Fribroug (Suisse), ainsi que directeur à Bellinzona, dans la Suisse italienne, de l'Instituto di Ricerche Economiche. Il est membre depuis sa fondation, du GREMI (Groupe de recherche européen sur les milieux innovateurs) et il a ainsi participé à toutes les enquêtes et publications de ce groupe. Il prépare actuellement un ouvrage sur « l'innovation technologique et le développement régional " (à paraître, IRE, 1992). Adresse : IRE, Stabile Torretta CH 6501, Bellinzona, Suisse. 


\begin{abstract}
This contribution analyses the evolution of the local and international relationships following different models of the spatial diffusion of innovation. The author tries, in an original fashion, to interpret the position of the innovative SME by distinguishing three life spaces of the firm: the market space, production space, and the supporting space.

The results show that, in a classical fashion, the SME will tend to act following a scheme defined by its market space and in a model where the rules of the game will be dictated by the market and by the urban hierarchy. In a structural context of the spatial division of work, on the contrary, the SME will, above all, act in such a way to adapt its own "production space " without having to reinforce a certain subordination in its relationship with the large multinational enterprise and metropolitan centers.

Finally, the analysis following a net-like model will, theoretically, place the SME in a position of reinforcement in relation to the already mentioned subordinations. This holds true, however, only if the enterprise knows how to develop its "supporting space", that is, the strategy of relationship "outside the market". It is important to note that this supporting space will be most likely attainable in certain places spatially marked by a new cohesion, by some local synergies being as well a constitutive element and a result of the existence of an "innovative system" following the GREMI terminology.
\end{abstract}

\title{
RESUMEN
}

La presente contribución analiza la evolución de las relaciones locales $e$ internacionales según diferentes modelos de la difusión espacial de la innovación. El autor intenta, de un modo original, interpretar la posición de la PME innovadora, diferenciando tres espacios de vida de la empresa; el espacio de mercado, el espacio de producción y el espacio de apoyo.

Los resultados nos indican que, desde un punto de vista clásico, la PME se comportará siguiendo un esquema definido por su espacio de mercado y por un modelo donde las reglas de juego las impondrá el mercado y la jerarquía urbana. De otra parte, en un contexto estructural de la división espacial del trabajo, la PME tendrá que adaptarse a su “ espacio de producción », sin tener que mantener una cierta subordinación en su relación con la gran empresa multinacional y los centros metropolitanos.

Finalmente, el modelo de análisis reciente, según un enfoque reticular, sitúa teóricamente a la PME en una posición de liberación respecto a la subordinaciones citadas anteriomente. Esto se consigue, solo si la empresa conoce como desarrollar su "espacio de apoyo", es decir la estrategia de relación "fuera del mercado". Es importante destacar que éste espacio de apoyo será posible en algunos lugares espacialmente representados por una nueva cohesión, por algunas "synergies „locales que integran un elemento constitutivo y un resultado de la existencia de un "sistema innovador "según la terminología del GREMI. 


\section{Introduction}

Selon notre analyse de l'évolution du rapport local-international d'après les différents modèles (ou théories) de la diffusion spatiale de l'innovation, nous pouvons distinguer trois familles de modèles de diffusion: les modèles épidémico-hiérarchiques, les modèles de la division spatiale du travail, les modèles réticulaires. Nous avons aussi essayé, d'une façon originale, de comprendre la position de la PME innovatrice en distinguant trois espaces de vie de la firme: l'espace de marché, l'espace de production et l'espace de soutien. Ce dernier décrit trois types de relations " hors maché " : les relations qualifiées au plan de l'organisation des facteurs de production; les relations stratégiques de l'entreprise concernant ses partenaires; les relations stratégiques de l'entreprise avec les acteurs de l'environnement territorial.

Les résultats de l'analyse montrent que, dans une vision classique, la PME agirait plutôt selon le cadre défini par son espace de marché et dans un modèle où les règles du jeu seraient dictées par le marché et par la hiérarchie urbaine. Dans un contexte structurel de division spatiale du travail la PME devra, au contraire, surtout agir pour adapter son « espace de production » (standardisation de la production par segments technologiquement homogènes) sans pour autant s'affranchir d'une certaine subordination par rapport à la grande entreprise multinationale et aux centres métropolitains.

Enfin, le modèle récent d'analyse selon une approche réticulaire place théoriquement la PME dans une position d'affranchissement par rapport aux subordinations citées, à la condition qu'elle sache développer son espace de " soutien ", c'est-à-dire une stratégie de rapport « hors marché » à caractère précompétitif. Or, ce qui est intéressant, c'est que cet espace de soutien sera réalisable, selon une forte probabilité, dans certains lieux spatialement marqués par une cohésion nouvelle, par des synergies locales qui constituent à la fois un élément constitutif et un résultat de l'existence d'un «milieu innovateur », selon la terminologie du GREMI.

\section{Les " espaces stratégiques " de la PME locale et leur environnement spatio-fonctionnel}

Nous commençons notre raisonnement au niveau micro, celui de l'entreprise (PME), que nous voulons toutefois saisir d'emblée dans sa dimension spatiale. Une PME sera alors caractérisée : 
- Par sa localisation spécifique à l'intérieur d'un espace territorial ouvert, c'est-à-dire défini en termes d'espace relationnel. Ce dernier point est important, car il pose déjà le problème en termes relatifs et dynamiques : l'avenir d'une entreprise ou d'un ensemble d'entreprises appartenant à une région dépendra non seulement de sa force ou de son contenu, mais aussi de son espace relatif de position.

- Par la définition de toute une série de relations spatio-fonctionnelles concernant les éléments déterminants du processus du développement de l'entreprise. Ces espaces spatio-fonctionnels, nous les nommons : " espaces stratégiques de l'entreprise ".

Dans les espaces « stratégiques » ou « de vie » d'une entreprise, nous pouvons distinguer trois espaces fonctionnels ${ }^{1}$ :

- L'espace de production de la firme. Cet espace sera déterminé par la division spatiale du travail selon le modèle de la théorie des segments. Ainsi, une entreprise pourra acheter à l'extérieur, voire relocaliser sa production vers l'extérieur, selon les caractéristiques technologiques, économiques et socioculturelles propres à chaque segment et à chaque région de production.

- L'espace de marché. Il est défini par les relations de l'entreprise avec les marchés. Ces relations, qui doivent pouvoir être lues spatialement, seront caractérisées par le nombre, l'intensité, les caractéristiques structurelles, ainsi que par le processus d'évolution des marchés (par reférence donc à un environnement).

La définition en termes spatio-fonctionnels de l'offre et de la demande constituerait déjà un grand progrès dans l'approche dynamique du développement des entreprises. Toutefois, elle nous semble encore insuffisante pour saisir les aspects stratégiques intérieurs et extérieurs (en termes de pouvoir) de l'entreprise. Nous proposons alors de définir un troisième espace fonctionnel de l'entreprise :

- L'espace de soutien. Il décrit trois types de relations « hors marché $»^{2}$ :

- les relations qualifiées ou privilégiées au plan de l'organisation des facteurs de production (origine du capital, source d'information, savoir-faire technologique, liens particuliers à l'égard du capital humain) ;

1. Nous avons déjà utilisé cette démarche dans deux études empiriques, l'une pour un secteur des services, l'autre pour analyser l'enquête GREMI II (Ratti et D'Ambrogio, 1988).

2. L'expression « hors marché » est le fruit d'un commentaire que R. Gordon nous avait adressé lors de la discussion au séminaire GREMI II, d'Ascona, 1988. 
- les relations stratégiques de l'entreprise concernant ses partenaires, fournisseurs ou clients (échange privilégié d'informations, collaboration, partenariat, alliance, intégration partielle) ;

- les relations stratégiques avec les acteurs de l'environnement territorial (institutions publiques, associations privées ou semi-publiques).

Nous pouvons représenter dans la figure 1 les traits caractéristiques des relations spatio-fonctionnelles d'une entreprise selon le modele a-spatial traditionnel et selon notre modèle définissant les espaces stratégiques de l'entreprise.

Comme on peut l'entrevoir dans notre schéma, notre troisième espace fonctionnel, l'espace de soutien, réalise et détermine en grande partie la relation entre l'offre et la demande, entre l'espace de production et l'espace de marché. Une bonne partie de ces relations sont intégrées dans l'espace de soutien, c'està-dire qu'elles précèdent les mécanismes de décision par les prix qui, en dernière analyse, sanctionneront le succès ou l'échec d'une production déterminée.

Pour comprendre les espaces stratégiques de l'entreprise, et notamment ce troisième espace, l'espace de soutien, il faut se référer à une approche de système. L'entreprise ne peut exister et survivre qu'à condition d'être capable de gérer ses relations avec l'extérieur. Ce pouvoir sera la résultante de la capacité de combiner ses ressources intérieures avec les flux exogènes, tout en considérant un environnement qui, continuellement, la conditionne dans son espace de position.

Sur le plan de l'analyse, l'étude des espaces stratégiques d'une entreprise passe alors par une rigoureuse détection des éléments déterminants du système, lesquels doivent être clairement établis et perçus dans leur fonctionnement à travers leurs rapports avec les autres éléments, toujours en lutte entre deux forces constitutives, une force centripète et une force centrifuge. Il s'agit d'emblée d'un processus dynamique, où le résultat donnera toujours un équilibre contingent, précaire, évolutif.

\section{La création de synergies locales}

Le passage de ce type d'analyse micro-spatiale de l'entreprise à un niveau des espaces méso-territoriaux pose naturellement deux types de problèmes : celui de l'agrégation sectorielle et celui des rapports entre espaces fonctionnels et espaces territoriaux (Crevoisier, 1988). S'il est vrai que chaque entreprise définit ses propres espaces de vie (de marché, de production et de soutien) à caractère fonctionnel, il devrait être cependant possible, voire nécessaire, dans l'optique 
de l'analyse de système, de passer à une agrégation des résultats au niveau des entreprises. Celle-ci semble heureusement possible grâce à la logique d'analyse de système, qui nous oblige non seulement à énumérer et à localiser les relations spatiales de la firme, mais aussi à qualifier et à structurer le type de relations de production, de marché, ou d'organisation stratégique (soutien) de l'entreprise. Ainsi, les relations d'une entreprise avec ses fournisseurs ou ses clients peuvent être simplement guidées par les rapports de "marché », ou bien aller, d'une façon plus ou moins structurée, au-delà du rapport de collaboration et atteindre celui de partenariat ou même une domination structurée par le biais d'une filiale.

Or, l'opération d'agrégation peut se révéler ici extrêmement intéressante, parce qu'elle fera apparaître ou non des synergies sectorielles et intersectorielles qui se manifesteront surtout, dans notre type d'analyse spatiale, par l'apparition de relations significativement structurées de la division spatiale du travail ; par exemple, la trame du réseau pourrait être un indicateur d'industrialisation diffuse ou, au contraire, de polarisation industrielle régionale, ou de division spatiale par segments ou, encore, de fragmentation et de désintégration des grandes firmes.

Le deuxième problème - celui des rapports entre espaces fonctionnels et espaces territoriaux - provient du fait que l'analyse spatio-fonctionnelle nous portera à définir une géographie de relations et de processus de développement qui ne correspondra pas ou, mieux, qui ne respectera pas les caractéristiques de continuité des espaces territoriaux-institutionnels (figure 2). Les relations fonctionnelles se développent (modèle actuel) avec des caractéristiques de forte verticalité, traversant des espaces institutionnels. Le « vertical » domine ou place « hors jeu » la dimension horizontale. Évoquons seulement la typologie des relations d'une grande entreprise multinationale ou celle qui est déterminée par un processus de segmentation et de division spatiale du travail.

La logique des relations spatiales au niveau fonctionnel peut toutefois, avec une certaine probabilité, donner lieu à des relations spatiales non indifférenciées et déterminer des espaces fonctionnels " polarisés » ou des espaces fonctionnels « champs de force ${ }^{3}$ ». Or, selon les caractéristiques géographiques des polarisations ou des «champs de force » fonctionnels, on peut reconduire, mais dans certains cas uniquement, une partie essentielle de l'espace fonctionnel vers l'espace territorial. La dimension horizontale serait alors récupérée : la dynamique des espaces de soutien pousse les entreprises soit à rechercher des intérêts communs, soit à collaborer avec les institutions territoriales pour la création d'un environnement favorable. Cette relative coöncidence

3. Selon la terminologie de G. Dellaporta (1963), appliquée par R. Ratti (1980). 
FIGURE 1

Les espaces stratégiques économico-fonctionnels de l'entreprise

Modèle traditionnel a-spatial

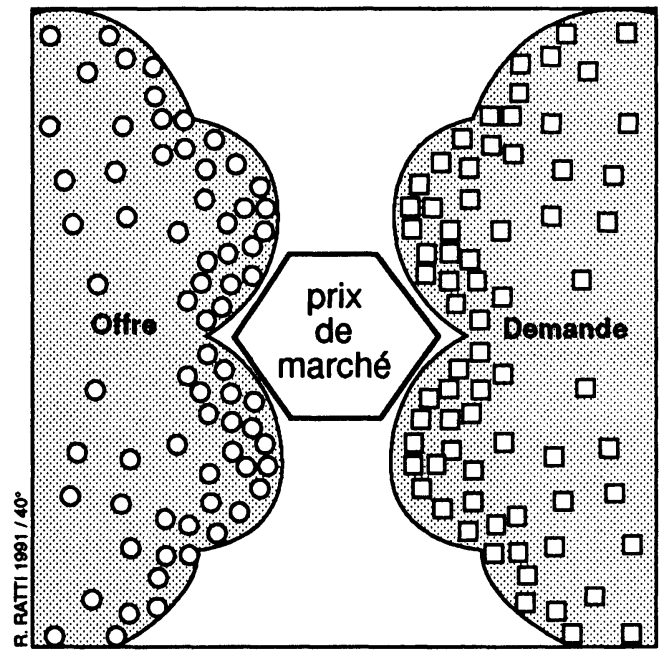

Légende: $O$ offreurs génériques $\square$ clients génériques

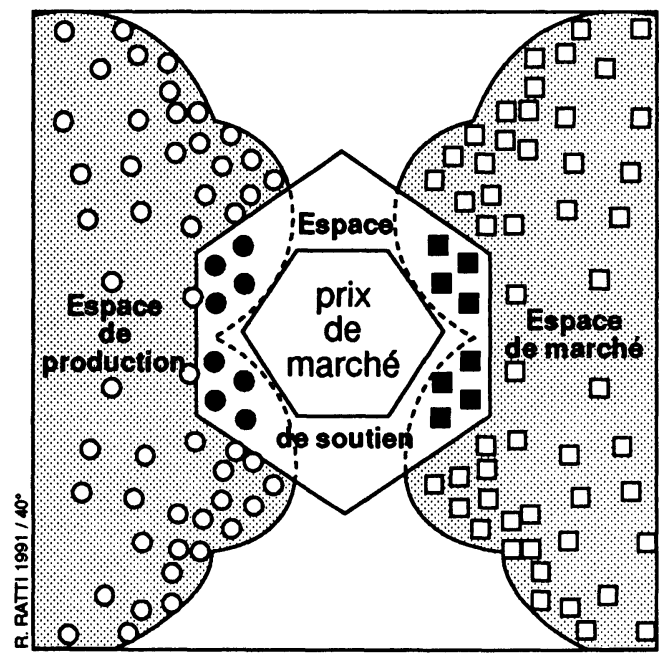

Légende: Ooffreurs génériques $\square$ clients génériques - offreurs ou

clients, en relation stratégique qualifiée avec l'entreprise 
entre un noyau fort des espaces fonctionnels et des espaces territoriaux nous permet alors de parler de « synergies locales » et de les définir comme le résultat d'une matérialisation territorialisable d'un ensemble de relations fonctionnelles structurées en termes de pôle ou de champs de force. Observons que cette logique nous conduit vers d'autres notions utilisées dans la littérature régionale récente, telle que celle de « tissu industriel local », d' " écosystème localisé », de " district industriel », de "système industriel localisé » et, pour finir, vers la notion de « milieu », développée par le GREMI $(1985 ; 1988)$.

Pour rester dans le cadre du titre et des objectifs de cette contribution, nous voyons alors les synergies locales comme l'un des facteurs déterminants, mais partiel, du milieu. En effet, notre démarche analytique nous porte vers les éléments constitutifs du milieu, c'est-à-dire "un processus de perception, de compréhension et d'action continuelle " où "la cohérence entre les différents acteurs réside dans une manière commune d'appréhender les situations, les problèmes et les opportunités » (Crevoisier, 1988).

FIGURE 2

Rapport entre espaces fonctionnels des entreprises et espaces territoriaux-institutionnels

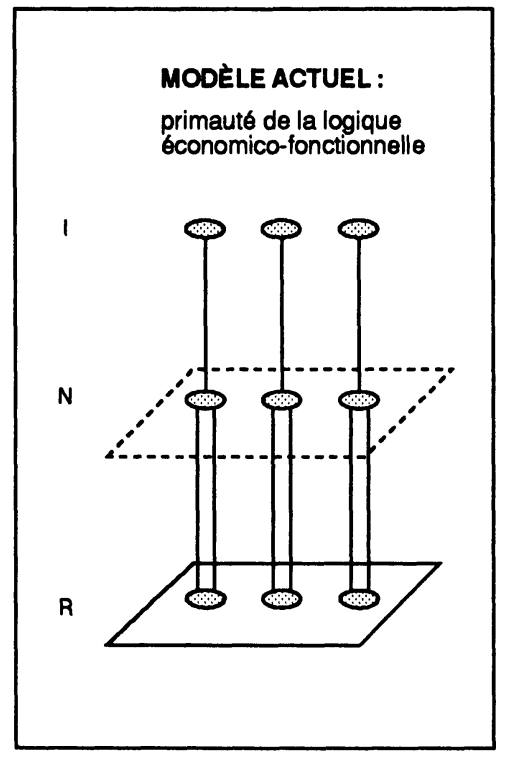

Source : R. Ratti (1991).

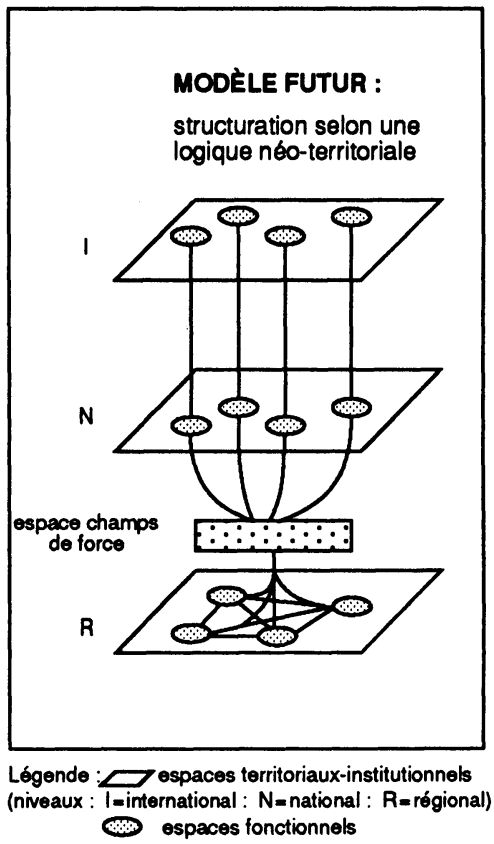

Revue internationale P.M.E., vol. 4, $\mathrm{n}^{\circ}$ 3, 1991 
En conclusion, parmi les espaces stratégiques de l'entreprise que nous avons définis, l'espace de soutien apparait sous une double lumière : en tant qu'élément constitutif et en tant que résultat de l'existence d'un milieu. Selon les canons de l'analyse systémique, cet espace fonctionnel de soutien - qui peut s'exprimer en tant que pôle ou champ de force - est essentiellement dynamique et en équilibre précaire entre force intérieure et force extérieure.

\section{Le rôle des synergies locales dans les processus spatiaux d'innovation et leur impact sur l'évolution du rapport local/international}

À l'aide des différents modèles de la diffusion spatiale de l'innovation (voir annexe), il apparaît intéressant de voir comment, dans chacun des cas, situer l'entreprise - en particulier la petite et moyenne (PME) - , et voir, à l'aide de notre typologie des espaces de vie de l'entreprise, quelles sont les réponses stratégiques qui s'offrent à l'entreprise. Nous pouvons alors l'exprimer sous forme de thèses :

\section{Thèse 1 Le rôle du milleu (environnement local) dans les processus d'innovation des PME varie selon les différents paradigmes de la diffusion spatiale de l'innovation}

On peut relever les points suivants :

- Le modele épidémico-hiérarchique nécessite une adaptation stratégique de l'entreprise qui suivra plutôt l'évolution de la demande et des marchés. Dans ce cas, les règles du "marché » et les caractéristiques de la hiérarchie urbaine détermineront aussi les conditions de localisation de la production (espace de production), offriront aux entreprises les économies d'agglomération et définiront l'espace de soutien. Cet espace de soutien aurait toutefois pour caractéristique d'être fourni par l'environnement au lieu d'être activement recherché par l'acteur-PME.

- Dans le cas des modeles de division spatiale du travail, les modalités techniques et stratégiques de la production semblent être déterminantes. Le moteur viendra des grandes entreprises multinationales (GEM) ou des pôles « core areas »; les espaces de marché des entreprises, ou, mieux, la diffusion spatiale de la production (surtout dans le cas d' « innovation radicale») se fera graduellement suivant la maturité du produit (théorie spatiale du cycle du produit). Aussi, pour les GEM, 
l'espace de soutien sera-t-il déterminé par la capacité d'intégrer et de gérer globalement un système de relations complexes (internationalisation), tandis que pour les PME, l'espace de soutien sera un espace conditionné par la stratégie de la GEM et limité aux caractéristiques socioculturelles de la région de localisation. Selon la terminologie de Aydalot (1985), les centres, ou la grande firme, jouent sur le « décalage » entre forme de production et modes de vie : à la périphérie, les modes de vie seront toujours en retard par rapport à la culture industrielle des formes de production conçues au centre ; ainsi, les petites et moyennes entreprises décentralisées ne pourront que s'appuyer sur un espace de soutien limité et insuffisant pour affronter à armes égales des impulsions et des forces venant du centre.

FIGURE 3

Matrice des comportements stratégiques (CS) des entreprises innovatrices selon les différents modèles de diffusion spatiale de l'innovation

\begin{tabular}{|c|c|c|c|}
\hline $\begin{array}{l}\text { Modèles des } \\
\text { diffusion } \\
\text { spatiale de } \\
\text { l'innovation }\end{array}$ & $\begin{array}{c}\text { Modèle } \\
\text { "Épidémico- } \\
\text { hiérarchique " }\end{array}$ & $\begin{array}{l}\text { Modèle } \\
\text { "division } \\
\text { spatiale du } \\
\text { travail " }\end{array}$ & $\begin{array}{c}\text { Modèle } \\
\text { " réticulaire " }\end{array}$ \\
\hline $\begin{array}{c}\text { Espace de } \\
\text { marché } \\
\text { (EM) }\end{array}$ & $\begin{array}{l}\text { Le CS est } \\
\text { fortement } \\
\text { déterminé par } \\
\text { l'EM } \\
\text { ( cas d'innovation } \\
\text { adaptive ) }\end{array}$ & $\begin{array}{l}\text { L'EM est subor- } \\
\text { donné à la logique } \\
\text { de la division } \\
\text { spatiale du travail }\end{array}$ & $\begin{array}{l}\text { L'EM aura souvent } \\
\text { les caractéristiques } \\
\text { du « marché } \\
\text { de niche " ou } \\
\text { de sous-traitance } \\
\text { qualifiée }\end{array}$ \\
\hline $\begin{array}{l}\text { Espace de } \\
\text { "production" } \\
\text { (EP) }\end{array}$ & $\begin{array}{l}\text { Les caractéristiques } \\
\text { de l'a EP " seront } \\
\text { plutôt } \\
\text { subordonnées à } \\
\text { l'“ EM" }\end{array}$ & $\begin{array}{l}\text { Le CS se joue en } \\
\text { bonne partie à ce } \\
\text { niveau ( innovation } \\
\text { radicale pour les } \\
\text { GEM ; localisation } \\
\text { des établissements } \\
\text { PME }\end{array}$ & $\begin{array}{l}\text { Forte spécialisation } \\
\text { et intégration des } \\
\text { espaces } \\
\text { de production }\end{array}$ \\
\hline $\begin{array}{l}\text { Espace de } \\
\text { "soution " } \\
\text { (ES) }\end{array}$ & $\begin{array}{l}\text { "ES " est } \\
\text { implicitement } \\
\text { reconduisible aux } \\
\text { différents lieux de la } \\
\text { hiérarchie urbaine }\end{array}$ & $\begin{array}{l}\text { Dans le cas de GEM } \\
\text { l'“ ES " aura un } \\
\text { caractère a-spatial } \\
\text { parce qu'inter. } \\
\text { nationalisé. Pour } \\
\text { les PME l'“ ES" } \\
\text { sera faible ou } \\
\text { subordonné }\end{array}$ & $\begin{array}{l}\text { La capacité d'inté- } \\
\text { gration réseau/ } \\
\text { milieu est détermi- } \\
\text { nante pour } \\
\text { l'innovation } \\
\text { adaptative/ } \\
\text { incrémentale } \\
\text { de la PME }\end{array}$ \\
\hline
\end{tabular}


- les hypothèses sur lesquelles est fondé un modele réticulaire permettent au contraire une meilleure, voire nécessaire, caractérisation de l'entreprise. À l'intérieur de ce modèle, l'espace de soutien prend le dessus par rapport aux deux autres espaces de vie de l'entreprise, l'espace de marché et l'espace de production. En effet, la nécessité de définir et de gérer des coopérations devient vitale, surtout pour une « innovation graduelle » (« incrémentale »). Ce type de coopération peut être subdivisé en trois grandes catégories :

- la première concerne une cooperation verticale et les relations du type « vendeur-acheteur »;

- la deuxième catégorie a trait à la coopération «horizontale et compétitive » entre des entreprises qui, tout en étant fondamentalement concurrentes, essaient de trouver des points d'avantages réciproques ;

- enfin, la troisième catégorie vise la coopération horizontale entre entreprises complémentaires. La complémentarité souvent se manifeste par le fait qu'un client emploie des produits de différents fournisseurs.

En général, cette coopération se fait en garantissant l'identité réciproque des acteurs et les cas de diffusion ou d'annexion auraient plutôt un effet pénalisant sur la stimulation des échanges (baisse de tension relative).

\section{Thèse 2 La centralité des synergles locales et la nouvelle dimension du rapport local/international}

À ce stade du raisonnement, on peut remarquer le potentiel explicatif de la conjugaison des deux éléments les plus originaux de notre construction: la référence à un modele réticulaire et la définition d'un "espace de soutien ». Si, dans le modèle épidémico-hiérarchique, la firme trouve son avantage dans les économies externes liées à une localisation selon la hiérarchie urbaine et si, dans le modèle de division spatiale du travail, le moteur de diffusion spatiale est déterminé par le captage de la "plus-value extra » basée sur une localisation périphérique, dans notre dernier cas, les localisations les plus favorables à la firme innovatrice seront déterminées par les lieux où l'entreprise peut construire le plus facilement son espace de soutien en termes d'économie externe d'organisation et d'information surtout.

Or, ce type d'économies, qui en principe ne se réfère pas à un espace nécessairement contigu, a une bonne probabilité de se territorialiser, c'est-à-dire de se matérialiser dans un espace concret en termes de synergies locales, voire de «milieux». En effet, l'espace de soutien sera d'autant plus facilement 
réalisable qu'il recourt aux facteurs d'innovation à caractère territorial déjà cités (Aydalot) et sera reconductible selon les trois catégories suivantes : nature du tissu industriel local ; facteurs d'attraction ; facteurs de synergie.

Les synergies locales se trouvent alors au centre de l'explication des dynamiques industrielles. En plus, dans sa représentation idéale, le caractère stratégique et volontaire de l'espace de soutien permet aussi de dépasser le traditionnel rapport d'infériorité implicite dans la liaison entre le local et l'international. Dans une approche réticulaire, la primauté des stratégies dites de " soutien " - c'est-à-dire des relations hors marché ou précédant le marché permet un affranchissement relatif des traditionnels rapports de subordination de la PME ou de l'entreprise localisée à la périphérie. Les probabilités d'affranchissement seront plus fortes dans le cas d' « innovation graduelle » et en présence d'un milieu bien différencié et ouvert.

\section{Thèse 3 Le rôle du milieu (environnement local) dans les processus d'innovation des PME dépend du type d'Innovation}

Le rôle des synergies locales dans le rapport local/international tend à varier selon la nautre de l'innovation (radicale, adaptative ou de perfectionnement). La figure 4 donne un exemple synthétique de la relation entre le type d'innovation, le comportement stratégique de l'entreprise et le milieu. L'innovation radicale demande plutôt une référence à un espace stratégique de production qui, à son tour, ne se laisse pas facilement identifier avec un «milieu » territorialement délimité. Au contraire, l'innovation adaptative demande l'existence d'une certaine polarisation de la production et des marchés. Enfin, l'innovation de perfectionnement est celle qui bénéficierait le plus facilement de l'existence d'un « milieu » spécialisé, voire intégré, et ouvert aux dynamiques extérieures.

\section{Conclusions dans l'optique de la politique régionale}

Dans une optique de politique régionale, soulignons que, même dans le modèle réticulaire, les conditions de développement régional ne seront pas nécessairement orientées vers le rééquilibrage. Certes, le processus de développement est proche du paradigme nouveau du développement territorial endogène. Toutefois, les prémisses de développement ne seront pas données partout. Il reste à rassembler et à faire interagir les facteurs qui déterminent les synergies locales et les propriétés d'un " milieu ». La dynamique et le rôle observés au plan de l'espace de soutien des entreprises montrent toutefois que les entreprises peuvent se libérer elles-mêmes de la logique purement fonctionnelle et sectorielle 
et chercher un meilleur avantage dans des synergies locales qui sont en même temps un facteur et un produit du «milieu». Or, cette orientation doit être doublée - sauf dans les rares cas de phénomène spontané - par une action volontariste au niveau des institutions territoriales (ainsi que nous l'avons évoqué dans la figure 2). En d'autres termes, une politique de développement (Aydalot, 1987) qui considérerait les espaces doit se préoccuper soit d'une lecture globale et systémique des espaces fonctionnels des entreprises, soit d'un encadrement des décisions susceptibles d'avoir un impact hiérarchisé sur la structure spatiale ; cela, afin de favoriser une meilleure mise en valeur du potentiel régional, en tant que sous-ensemble d'une économie de plus en plus mondiale.

FIGURE 4

Analyse dynamique des liaisons « innovation - espaces stratégiques - milieu »

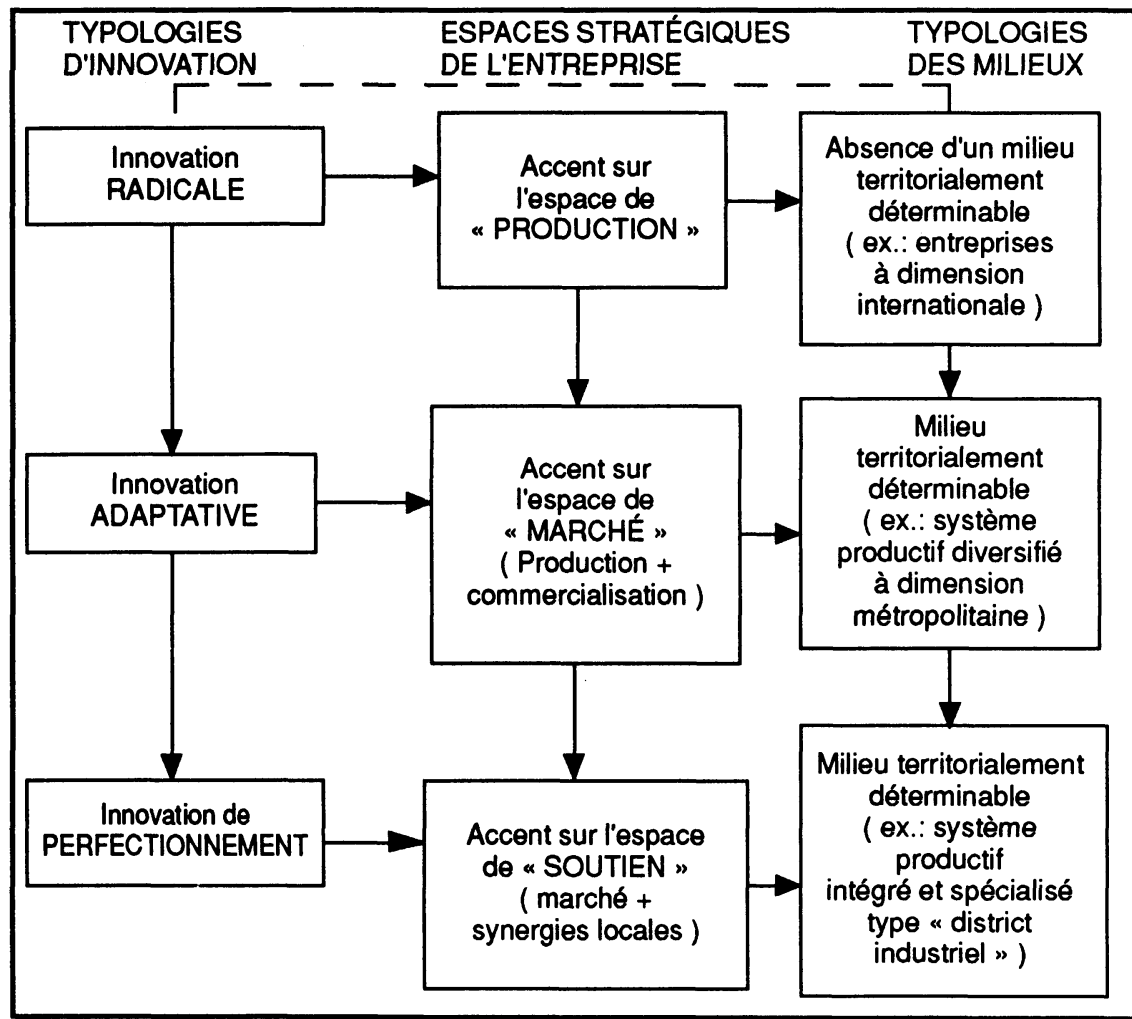

Source : GREMI II (Bramanti et Senn, 1989) 


\section{Annexe : Les différents modèles de la diffusion spatiale des innovations}

\section{Le modèle épidémico-hiérarchique}

Selon ce modèle, la diffusion spatiale des innovations peut suivre différents canaux :

- Elle peut se faire par contagion selon le critère de voisinage. Pour cette raison, on a parlé de "théorie épidémique de la diffusion des innovations ». Ce processus se fait selon une loi de probabilité de contact, diminuant avec la distance, notons qu'alors la diffusion ne sera pas homogene (importance de voies de communication des obstacles). L'adoption de l'innovation se ferait dans le temps selon une courbe logistique, tandis que dans l'espace, cette adoption serait en fonction décroissante de la distance.

- Elle peut se faire suivant les canaux de la hiérarchie urbaine.

En effet, le degré de proximité de deux lieux dépend, non seulement de leur distance dans l'espace physique, mais aussi de la position relative qu'occupent ces lieux dans l'organisation du territoire. Le système des lieux centraux offre donc des canaux privilégiés de propagation. Une grande ville aura des probabilités plus grandes de devenir un centre émetteur puissant qu'une petite. Ainsi, les probabilités que le message soit transmis, que le contact ait lieu et que l'innovation soit diffusee sont beaucoup plus importantes de la grande vers la petite ville, du sommet vers le bas de la hiérarchie urbaine qu'en sens inverse (SaintJulien, 1985). Cette approche reliée à l'apport géographique peut être complétée par des considérations économiques plus spécifiques selon la théorie dite du «filtering down»:

- Le milieu urbain, et plus précisément le pôle urbain, présente des caractéristiques positives qui limitent le degré d'incertitude face aux innovations.

- Tout au début, dans la phase expérimentale, une localisation dans le pôle peut être idéale, mais par la suite une centralisation ne serait plus toujours efficiente (coûts de congestion, coûts de main-d'œuvre, nécessité d'être près du marché) ; c'est ainsi que le pôle laisserait progressivement filtrer certaines activités attribuées aux éléments inférieurs de la hiérarchie urbaine (Camagni et Cappellin, 1984). 
FIGURE 5

La diffusion spatiale de l'innovation

selon le modèle épidémico-hiérarchique

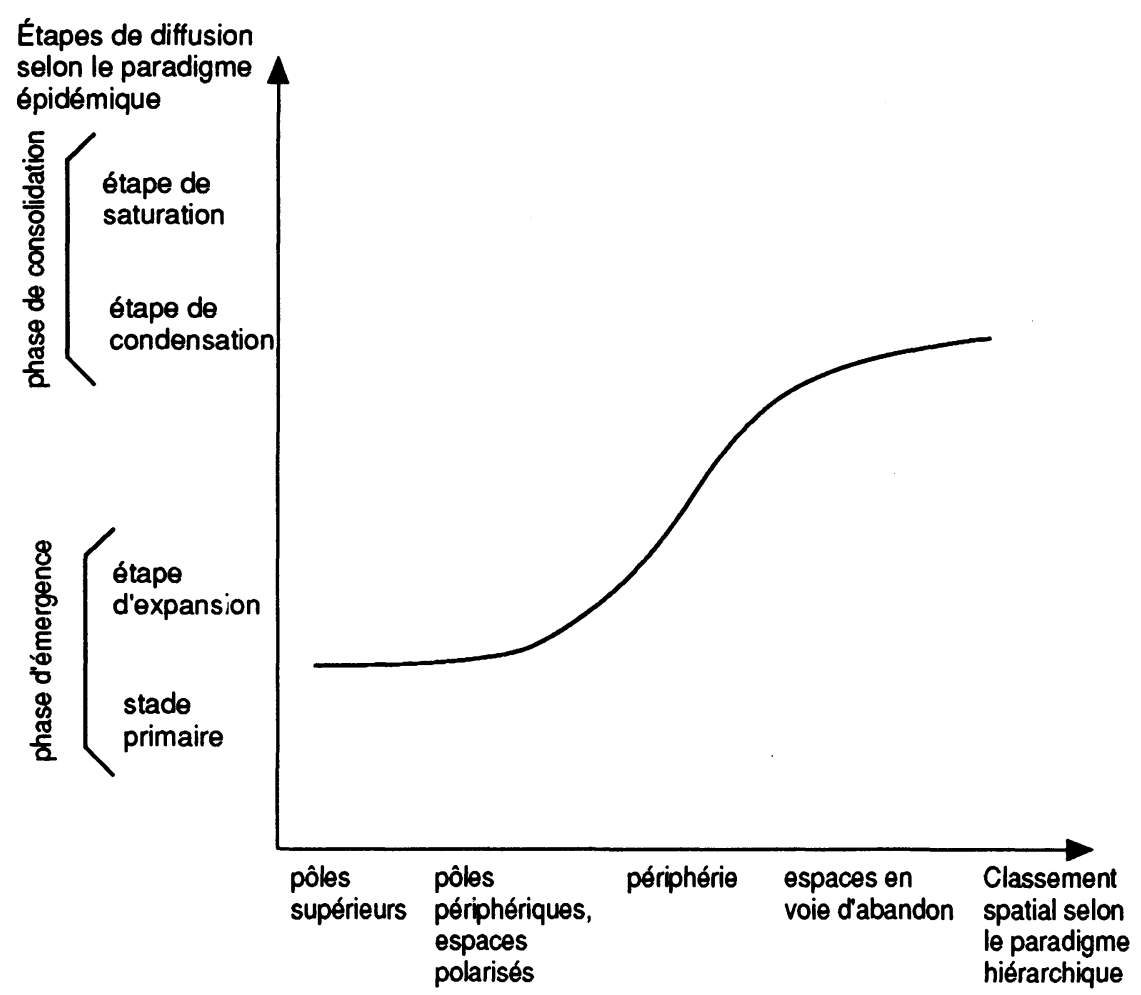

Source : R. Ratti (1991).

\section{La diffusion selon les modèles de la division spatiale du travail}

Cette formulation, qui date de la fin des années 70 , tente d'intégrer la formation des processus centre-périphérie dans une conception d'ensemble de l'espace et du développement régional (OCDE, 1986). Selon cette formulation, il y a division spatiale du travail - et aussi une division spatiale de l'innovation - parce que la grande entreprise surtout est capable de scinder l'ensemble de son activité en unités aussi homogènes que possible selon le type de travail auquel ces centres recourent, et de localiser et de disperser ces unités dans les lieux les plus favorables en termes de travail et de culture industrielle. Ce résultat serait 
le fruit d'un processus de diffusion des activités sur le plan mondial qui ne suit pas la hiérarchie spatiale traditionnelle, mais qui est déterminée par une hiérarchie fonctionnelle propre aux grandes entreprises multinationales. La diffusion technologique est ici déterminée par l'offre, c'est-à-dire par la stratégie interne de la GE. La mobilité accrue est facilitée par les progrès en matière de transports et de communication, ainsi que par la capacité de standardisation des techniques, même les plus sophistiquées, qui permettraient d'obtenir une productivité suffisante avec un travail peu qualifié. Cette démarche permet aussi de développer les marchés par la diffusion des modes de vie "centraux ». Cela oblige toutefois à un élargissement permanent de l'espace bien que, avec le démarrage de technologies nouvelles, le processus comporte une phase de repolarisation. Toutefois, la phase de repolarisation n'est que provisoire et devient de plus en plus courte (Silicon Valley) et peut concerner aussi des lieux nouveaux (Midi de la France, Floride). C'est ainsi que la réalité spatiale présentera une typologie d'évolution complexe : un pôle devient centre (limité à la fonction de direction) et peut, successivement, redevenir pôle, rester centre (New York) ou être abandonné (crise de régions d'ancienne industrialisation).

Remarquons pour terminer que la logique de cette approche va au-delà de l'analyse en termes de coût de production ou de cycle de vie du produit. En effet, selon P. Aydalot, l'élément central qui déterminerait cette hiérarchie spatiale se trouve dans les décalages entre les formes de la production et les modes de vie. Ces derniers s'adaptent, mais seulement avec une certaine inertie, aux modèles sociaux promus par une nouvelle technologie. "Lorsqu'une technologie nouvelle est mise en action dans un milieu dont le fonctionnement est encore déterminé par des formes techniques antérieures, le décalage ainsi créé peut être porteur d'une plus-value pour l'entreprise puisque le coût de reproduction de la force de travail est encore calqué sur les pratiques de production antérieure tandis que la productivité s'est accrue » (Aydalot, 1985).

\section{La diffusion spatiale de l'innovation selon le modèle "réticulaire " (milieu/réseau)}

Selon le Suédois Håkansson, la perspective dominante dans l'innovation et le développement technique se caractérise par le "syndrome de Newton » (Håkansson, 1987). En effet, l'innovation a toujours été représentée comme un fait individuel (par exemple, un "prix Nobel») ou comme un produit secret d'une entreprise. L'auteur de l'ouvrage cité propose une tout autre approche, laquelle voit l'innovation et le développement technologique principalement comme le fruit d'un échange entre différents acteurs, c'est-à-dire, comme le produit d'un « réseau». Or, les études empiriques, notamment au sein du 
GREMI même, nous démontrent le bien-fondé de cette approche réticulaire, qui, à certaines conditions seulement, est à l'origine et détermine un processus de développement technologique. On peut présumer des caractéristiques de cette approche par les considérations suivantes :

- Un réseau est constitué de trois classes de variables de base : les acteurs (ceux qui gèrent des activités ou contrôlent des ressources), les " activités » (divisibles en deux catégories : de transformation ou de transaction), ainsi que les «ressources » (physiques, financières, humaines).

- Ces variables sont reliées entre elles et sont soumises à une double action : positive, lorsqu'une connexion en appelle d'autres ; négative, lorsqu'une connexion en empêche certaines autres. Ceci implique qu'il y aura en même temps des élements de cooperation et des éléments de conflit dans les relations entre acteurs, entre différentes activités et entre différentes ressources.

- La cohésion d'un réseau est déterminée par certaines forces spécifiques: une interdépendance fonctionnelle entre acteurs, activités et ressources ; une structure de pouvoir, en termes de capacité systématiquement développée pour gérer les différentes composantes du réseau ; une structure de connaissance, constituée par le capital d'expériences et de savoir acquis précédemment par les différents acteurs ; une structure temporelle, c'est-à-dire définie également en termes de développement historique du réseau (ce qui suppose une certaine stabilité face aux changements).

À ces trois facteurs, il faut en ajouter un quatrième, que nous allons privilégier : la structure spatiale du réseau.

Cette structure spatiale du réseau complétera d'ailleurs un réseau de relations fonctionnelles à l'activité de production avec un «environnement " plus large et complet. Les caractéristiques de la dimension spatiale du réseau peuvent être très différentes, allant de l'espace discontinu et dispersé vers un espace hiérarchisé, et jusqu'à un espace qualifié, contigu, en termes de régions ou de milieux. Nous retrouvons ici les premières conclusions des travaux du GREMI (Ayladot, 1986) qu'il vaut la peine de citer :

Selon l'époque, les comportements nationaux, la nature de la technologie nouvelle, celle-ci se localisera dans des types d'espaces bien différents; systématiquement, chaque région ne peut innover que selon un processus (ou deux) bien défini ; toute région n'a pas vocation à accueillir une technopole au dynamisme fondé sur la fertilisation croisée et le spin off. 


\section{Bibliographie}

AyDalot, Ph. (éd.) (1986), « Milieux innovateurs en Europe », Paris, GREMI, 1986, p. 359.

Aydalot, $\mathrm{Ph}$. (1987), «Le concept de région dans la planification régionale », dans Revue canadienne de science régionale, $\mathrm{X} 2, \mathrm{p} .213$.

Aydalot, $\mathrm{Ph}$. et $\mathrm{D}$. KeeBle (éd.) (1988), High Technology Industry and Innovative Environments : The European Experience, Londres et New York, GREMI.

Camagni et Cappeidin (1984), «Cambiamento tecnologico e diffusione territoriale », Milano, F. Angeli Editore.

CAMAGM (éd.) (1991), Innovation Networks, Londres et New York, GREMI, Belhaven Press.

CREvoisier, O. (1988), «Les logiques territoriales et fonctionnelles et leur articulation dans la région ", Colloque ARGO.

Della Porta, G. (1963), « Sviluppo economico regionale - teoria politica », Rocca San Cosciano, p. 34.

HÁkansson, H. (éd.) (1987), Industrial Technological Development - a Network Approach, Londres, Croom Helm, cop.

RATTI, R. (1980), «Investimento pubblico ed effetti economico-spaziali. Teoria ed applicazione nell'analisi costi-benfici », Frigourg, Éditions universitaires, Collection ISES, p. 79-87.

RATTI, R. (1989), « PME, synergies locales et cycles spatiaux d'innovation », Fribourg, cahier de recherche.

RATTI, R. (1991), " Small and medium-size enterprises, local synergies and spatial cycles of innovation », dans Camagni (éd.) Innovation Networks, Londres et New York, Belhaven Press.

RATT, R. et F. D'AMBRogio (1988), «Un essai d'analyse fonctionnelle et territoriale de l'industrie innovatrice au Tessin », Ascona, Colloque GREMI, (à paraître).

SAINT-JulieN, T. (1985), «La diffusion spatiale des innovations », Montpellier, GIP Reclus.

WADLEY, D. (1986), « Restructuring the Regions, analysis, policy model and prognosis », Paris, OCDE. 